\title{
Impacts of the invasive species Caulerpa cylindracea Sonder, 1845 on the algae flora of the west coast of Algeria
}

\author{
Benabdallah Bachir Bouiadjra ${ }^{\ddagger}$, Malika Ghellai§, Mohamed Daoudi ${ }^{\ddagger}$, Ibrahim Elkhalil Behmene ${ }^{\ddagger}$, \\ Mohammed El Amine Bachir Bouiadjra $\$$
}

‡ Laboratory of Animal Production Sciences and Techniques (LSTPA), University of Mostaganem, Mostaganem, Algeria

$\S$ University of Relizane, Relizane, Algeria

Corresponding author: Mohamed Daoudi (mohamed.daoudi@univ-mosta.dz)

Academic editor: Anne Thessen

Received: 17 Feb 2021 | Accepted: 10 May 2021 | Published: 26 May 2021

Citation: Bachir Bouiadjra B, Ghellai M, Daoudi M, Behmene IE, Bachir Bouiadjra MEA (2021) Impacts of the invasive species Caulerpa cylindracea Sonder, 1845 on the algae flora of the west coast of Algeria. Biodiversity Data Journal 9: e64535. https://doi.org/10.3897/BDJ.9.e64535

\begin{abstract}
The assessment of the impacts of the expansion of the invasive species on taxonomic diversity, the abundance and dominance of groups of algae, the presence and/or absence of species of ecological interest that may or may not be indicative of water quality well mentioned, through the installation of a $20 \times 20 \mathrm{~cm}$ quadrat representing the minimum area. The observation stations were visited monthly, during a repetitive three-year cycle, during the spring, summer and autumn seasons, periods of maximum growth and development of the algal flora and the results suggest the following facts. The invasive alga Caulerpa cylindracea Sonder, 1845 tends to colonise disturbed ecosystems reflecting a reduction in native algal diversity; in fact, we note a drastic impoverishment of the invaded algal community, represented by a limited number of Macrophyte algae accompanying the invasive taxon in phytosociological surveys and a Shannon-Weaver Diversity Index ( $\left.\mathrm{H}^{\prime}\right)$ and Equitability reduced by 4.49 and $0.77 \mathrm{n}$ the heavily affected station. The number of macroalgal species accompanying the invasive species has dropped by $52 \%$ in Salamandre. In addition, the multidimensional analysis, represented by the Hierarchical Ascendant Clustering applied to this case, confirms our results.
\end{abstract}




\section{Keywords}

algae, invasive, macrophytes, diversity, index, phytosociological, taxon

\section{Introduction}

Studies on ENI date back to the 1970s (Ojaveer et al. 2018). Since then, global research in this area has developed very rapidly (Giakoumi et al. 2019).

The Mediterranean Sea is today considered to be one of the most affected of the regional seas, due to the various anthropogenic pressures on the different ecosystems of the Mediterranean asin (Mannino et al. 2017). This favors it to be an important place for nonnative species, which can become invasive leading to a loss of biodiversity and changes in ecosystem services (Brunel et al. 2013, Giakoumi 2014).

The presence of mud-flats, estuaries and coral reefs along lagoons and rocky beaches provides ideal habitat for sustainable algae growth (Premarathna et al. 2019).

The concept of invasive species introduction is applied when the naturalisation of a species outside its usual geographic range is directly or indirectly linked to a human activity (Carlton 1985). The impact of introduced species has, thus, become the second most important cause of biodiversity loss in the world (Wilson 1997), after the outright disappearance of habitats. Bright (1998) defines the impact of introduced species on the planet's biodiversity as "evolution in reverse".

Amongst these bioinvasions, we are interested in Caulerpa cylindracea (Sonder 1845), recently reported on the Algerian west coast and which presents a strongly invasive behaviour during its colonisation (Manconi et al. 2020). It is considered to be one of the most threatening invasions of the Mediterranean Sea (Piazzi et al. 2016) and its impacts on algal biodiversity and seagrass beds are well reported in literature (Gribben et al. 2018, Montefalcone et al. 2015).

Indeed, the algal flora of the Algerian west coast remains largely unexplored. Direct observation of the algal settlement constituting the phytobenthos and its evolution is essential for monitoring possible changes in algal biodiversity. Therefore, the purpose of this research is to:

- establish a quantitative and qualitative inventory, located in time and space, of macrophyte species inhabiting two sites located on the coast of Mostaganem (west coast of Algeria Fig. 1), one affected by C. cylindracea, the other spare.

- $\quad$ assess the impact of invasive species expansion on taxonomic and specific diversity, abundance and dominance of algal groups, presence and/or absence of species of ecological interest that may or may not be indicative of water quality. 


\section{Material and methods}

The choice of harvesting stations was primarily based on the importance of the algal marine flora, the location of the stations studied shown in Fig. 1.

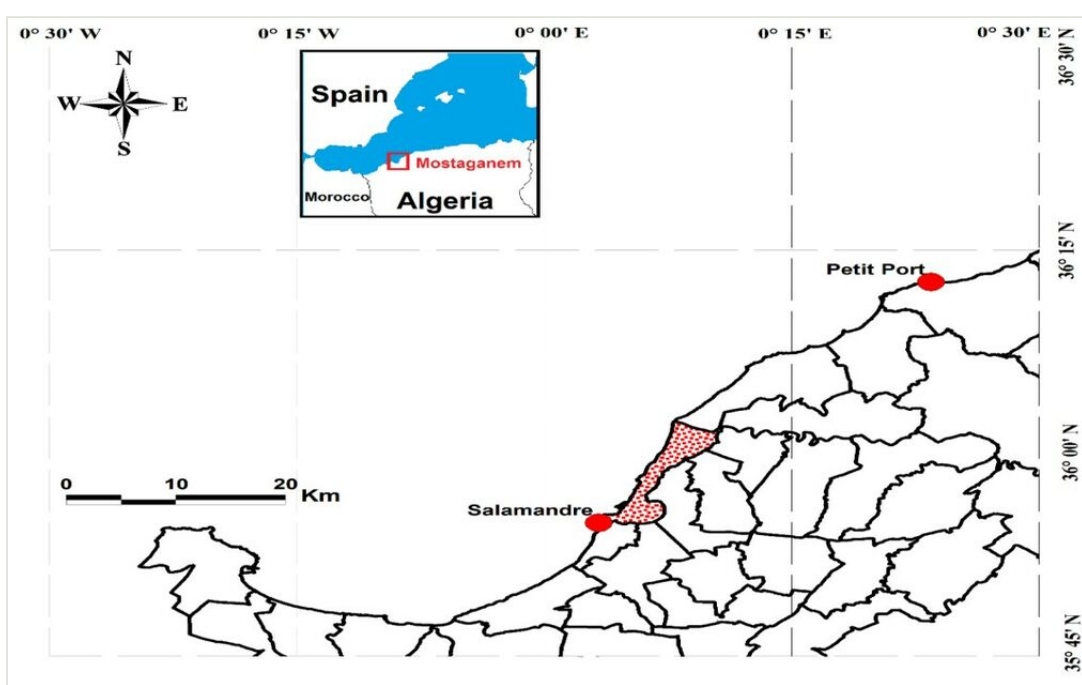

Figure 1. doi

The geographical location of the studied sites (Google Earth modified).

The area studied extends over $50 \mathrm{~km}$ of coastline, from Salamandre each to Sidi Lakhdar each (small port). Both stations were visited monthly during a repetitive cycle of three years, in spring, summer and autumn seasons, periods of maximum growth and development of the algal flora. In each station, we prospected a 50 to $100 \mathrm{~m}$ long and 10 to $15 \mathrm{~m}$ wide coastal line, with a depth of 5 to $10 \mathrm{~m}$.

Salamandre Station: The Salamandre site (Fig. 1) is $5 \mathrm{~km}$ from the town of Mostaganem, housing a new fishing port, its length around $4000 \mathrm{~m}$. Surveys conducted in sheltered, quiet areas within a rocky beach with the following coordinates: $35^{\circ} 55^{\prime} \mathrm{N} / 0^{\circ} 03^{\prime} \mathrm{E}$.

Small Port Station: This zone is located $35 \mathrm{~km}$ east of Mostaganem (Fig. 1), the sampling stations located in places with sandy and rocky bottoms on a beach $3500 \mathrm{~m}$ long sheltering a clear-seeded Posidonia oceanica meadow, associated with macrophyte algae with geographical coordinates, $36^{\circ} 12^{\prime} \mathrm{N} / 0^{\circ} 23^{\prime} \mathrm{E}$.

\section{Sampling, sorting and conservation of material.}

Sampling was performed on both soft and hard variable substrates. Sampling random, raking the rocks at each survey, three surveys per observation station and per year, according to climatological conditions and without biotope delimitation so that the sample is as representative as possible of the zone (Fig. 2). 


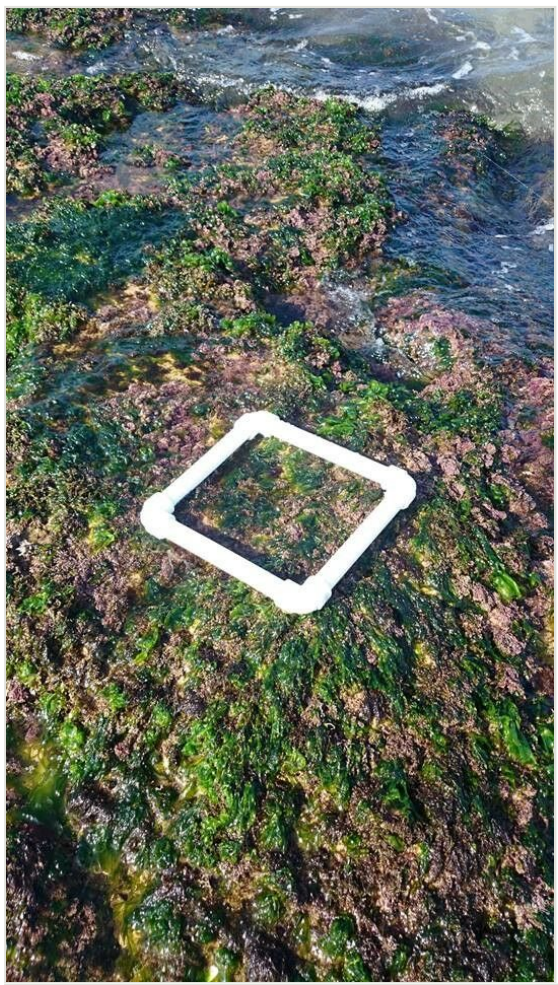

Figure 2. doi

Quadrat used for sampling $(20 \times 20 \mathrm{~cm})$.

\section{Species identification}

Different keys for the determination of macrophyte algal flora have been consulted see Fischer et al. 1987, Mojetta and Ghisotti 1996, Gayral 1958, Gayral 1966, Carrillo Marta Sanson 1999, Coppejans 1983, Hamel 1931, Hamel 1939, Verlaque 1990, Ballesteros et al. 2007, Boudouresque and Boudouresque 1969, Feldmann 1931, Feldmann 1933, Bliding 1968, Belhissoun 1995, allow us to draw up a catalogue which is by no means exhaustive of the species of algae inhabiting the two stations considered.

\section{Minimum Area}

The minimum area is the area where one has the best chance of finding all the species of the settlementstudied to within $10 \%$ according to Gounout (1969). For our case, the minimum area adopted is $20 \times 20 \mathrm{~cm}$ a surface of $400 \mathrm{~cm}^{2} \mathrm{Fig} .2$.

\section{Analytical Parameters}

For the quantitative and qualitative analysis of vegetation, we used the terrestrial phytosociological methods that several authors have adopted in the marine environment (Boudouresque 1971a, Boudouresque 1971b, Cormaci et al. 1997, Scammacca et al. 
1993). The results are expressed by their average, with data analysis carried out by the Statistica software (version 7.0) for all the parameters retained.

\section{Recovery}

The recovery $(\mathbf{R} \mathbf{i})$ is the approximate percentage of the substrate surface covered in projection by species $\mathbf{i}$. Given the stratification of vegetation, the total coverage of a survey $\sum \mathbf{R i}$ is generally greater than $100 \%$. The importance of recovery is expressed in class according to the following scale:

$+=$ negligible recovery

$1=$ less than $5 \%$ of the surface is covered

$2=$ between 5 and $25 \%$ of the surface area

$3=$ between 25 and $50 \%$ of the surface area

$4=$ between $50 \%$ and $75 \%$ of the surface area

$\mathbf{5}=$ more than $75 \%$ of the surface area

\section{Overall average recovery}

Each class of the recovery coefficient $\mathrm{Ri}$ is assigned a conventional monthly average value (class centre) called average recovery.

Absence $=0 ;+=01 \% ; 1=2.5 \% ; 2=15 \% ; 3=37.5 \% ; 4=62.5 \% ; 5=87.5 \%$

The RMG (overall average recovery) of species $\mathrm{i}$ in a set of $\mathrm{N}$ recorded is the average of these successive average recoveries.

\section{$\mathbf{R M G}=\sum \mathbf{R i} / \mathbf{N}$}

The RMG of a subset $E$ of $n$ species (ecological group, systematic unit or phytogeographic elements) is the sum of the constituent species.

\section{Species richness (Q).}

The $Q$ coefficient is the specific population size of any subset in a survey (ecological group, systematic unit or biogeographic features) and the $Q$ (highlighted) of a group of species representing its average species size.

\section{Quantitative dominance.}

The dominance based on recovery ( $\Sigma \mathrm{DRi}$ ) of a group of species in a survey (in a survey table), is the ratio, expressed as a percentage of the sum of their recovery (or their RMG) to the total recovery of species in the survey (or to the total average recovery of species in the survey table). 


\section{Qualitative dominance (DQ)}

This is the ratio expressed as a percentage of $Q$, (where $Q$ highlighted) to $T$ (where $T$ highlighted).

\section{Index of Specific Diversity (Shannon-Wiener)}

This ndex $\left(\mathrm{H}^{\prime}\right)$ measures, in a settlement, the amount of information resulting from species differentiation. The $\mathrm{H}^{\prime}$ value reflects the degree of structural evolution, maturity and stability of the ecosystem under consideration. It was calculated from the dominance of each species (Ri/Rt) according to Shannon's formula (in Boudouresque and Cinelli 1971).

$\mathbf{H}^{\prime}=-\sum \mathbf{P i}^{*} \log _{2} \mathbf{P i}$ with $\mathbf{P i}=\mathbf{R i} / \mathbf{R t} \mathbf{R t}=$ total recovery

he calculations were made from Suppl. materials 1, 2 under $R$ software version 3.0.3 $R$ Core Team 2014

\section{Evenness or Regularity E}

This is the ratio between the actual community diversity index and its maximum value for the number of species present $\mathbf{H}_{\max }=\log _{2} \mathbf{T}$ with $\mathbf{T}=$ total number of taxa

$E=H^{\prime} / H^{\prime} \max$

the calculations were made from Suppl. materials 1,2 under $R$ software version 3.0.3 $R$ Core Team 2014

\section{Taxonomic diversity}

Three taxonomic indices have also been adopted for the study of taxonomic diversity $\left(\Delta, \Delta^{*}\right.$ and $\left.\Delta^{+}\right)$. These were calculated from a taxonomic tree constructed on the basis of phylogenetic classification (Clarke and Warwick 1998), considering eight taxonomic levels: species, genus, family, order, class, phylum, under-reign and reign. From this tree, a taxonomic distance wij is quantified between each pair of species, assuming that this distance is equal to 100 for two species related to the highest taxonomic level considered in the study (Clarke and Warwick 1999). This calculated distance (wij) will then be integrated into the calculation of the various taxonomic indices. The calculations were performed using Suppl. materials 3, 1, 2 software R version 3.0.3 (R Core Team 2014). The phylogenetic classification is based on the Worms Editorial Board (Guiry and Guiry 2020).

With $x i(i=1, \ldots, S)$ : the abundance of the $i^{i t h}$ species, $N(=\Sigma x i)$ : the total number of individuals in the sample and wij: the distance to be covered between species $i$ and the first common node with species $\mathrm{j}$ in the hierarchical classification:

- $\Delta$ "Taxonomic diversity" (Warwick and Clarke 1995): 
$\Delta$ represents the average taxonomic distance between each pair of randomly selected individuals in the sample.

- $\Delta^{* " T a x o n o m i c ~ D i s t i n c t n e s s " ~(W a r w i c k ~ a n d ~ C l a r k e ~ 1995): ~}$

$\Delta^{*}$ is the average taxonomic distance between two randomly selected individuals belonging only to different species.

- $\Delta+$ "Average Taxonomic Distinctness", on data in presence/absence, $\Delta$ and $\Delta^{*}$ are simplified by $\Delta+$ (Clarke and Warwick 1998):

$\Delta^{+}$measures the average taxonomic distance between two randomly selected species.

\section{- Funnel Test:}

In order to detect assemblages whose taxonomic diversity would be influenced by disturbances, Warwick and Clarke (1998) proposed the "Funnel" test. This test consists comparing the taxonomic variety of a site to the area to which it belongs, from 1000 random draws of size $\mathrm{m}$ species made on the global list of $\mathrm{S}$ species listed in the area under consideration.

\section{Hierarchical Ascendant Clustering (HAC)}

The hierarchical classification has been used in a wide variety of disciplines. It has been described by several authors including Daget 1979, Dagnelie 1986, Legendre and Legendre 1984, Legendre and Legendre 1998, Herrera and Legac 2002 and Bouroche and Saporta 2005. Our study was confirmed by the use of this multidimensional analysis which consists in partitioning the objects (or descriptors) of the study into groups and subgroups, passing through the condensation of the information provided through the data matrix into a similarity or distance matrix. The latter will then be classified according to different classification algorithms (Ward, single, complete, average), which will each provide a corresponding dendrogram. The calculation procedure for this analysis was performed using Suppl. materials 1, 2 under software R version 3.0.3 (R Core Team 2014).

\section{Results}

Results are reported in Table 1 and Table 3 for algal stand composition at the Salamandre and Small Port Stations respectively and in Tables 2, 4 for analytical parameters (RMG, $\mathrm{DRi}, \mathrm{Q} D Q$ ) at the same stations. Table 5 represents the set of taxonomic indices at the two study stations and Fig. 3 illustrates the Funnel Test. The Hierarchical Ascendant Classification of the study stations based on the algae stands surveyed is illustrated in Fig. 4. 
Table 1.

Algal settlement sheet: Salamandre Station.

\begin{tabular}{|c|c|c|c|c|c|}
\hline Statement number & 1 & 2 & 3 & \multirow[t]{5}{*}{ RMG } & \multirow[t]{5}{*}{$\mathrm{Pr}$} \\
\hline Month & Mar. & Nov. & June & & \\
\hline Surface in $\mathrm{cm}^{2}$ & 400 & 400 & 400 & & \\
\hline Cover & $100 \%$ & $90 \%$ & $90 \%$ & & \\
\hline Exposition & $\mathrm{N}$ & NE & NW & & \\
\hline Erythrotrichia carnea & + & + & + & 0.10 & 3 \\
\hline Acrochaetium cheminii & 0 & + & + & 0.07 & 2 \\
\hline Colaconema daviesii & + & + & + & 0.10 & 3 \\
\hline Asparagopsis armata & 2 & 1 & 1 & 6.67 & 3 \\
\hline Falkenbergia rufolanosa & 1 & 1 & 1 & 2.50 & 3 \\
\hline Dasya rigidula & + & + & + & 0.10 & 3 \\
\hline Taenio mananum & 1 & 1 & 0 & 1.67 & 2 \\
\hline Chondria coerulescens & 0 & + & + & 0.07 & 2 \\
\hline Chondriamairei + C. capillaris & + & + & + & 0.10 & 3 \\
\hline Digenea simplex & 1 & 1 & + & 1.70 & 3 \\
\hline Halopithyin curvus & 0 & 1 & 1 & 1.67 & 2 \\
\hline Herposiphonia secunda f. secunda & 0 & 0 & + & 0.03 & 1 \\
\hline Herposiphonia secunda f. tenella & 0 & + & 0 & 0.03 & 1 \\
\hline Lophocladia lallemandii & + & + & 0 & 0.07 & 2 \\
\hline Vertebrata fruticulosa & + & + & + & 0.10 & 3 \\
\hline Vertebrata furcellata & 0 & + & + & 0.07 & 2 \\
\hline Polysiphonia spinulosa & 0 & 0 & + & 0.03 & 1 \\
\hline Amphiroa rigida & 2 & 1 & + & 5.87 & 3 \\
\hline Ellisolandia elongata & 2 & 2 & 2 & 15.00 & 3 \\
\hline Corallina officinalis & 0 & 2 & 1 & 5.83 & 2 \\
\hline Jania rubens & + & 1 & 1 & 1.70 & 3 \\
\hline Hypnea musciformis & 1 & 1 & 1 & 2.50 & 3 \\
\hline Grateloupia filicina & 0 & + & + & 0.07 & 2 \\
\hline Gastroclonium clavatum & 0 & + & + & 0.07 & 2 \\
\hline Dictyopteris polypodioides & + & + & 1 & 0.90 & 3 \\
\hline Dictyopteris divaricata & + & 1 & 1 & 1.70 & 3 \\
\hline Asperococcus bullosus & 0 & 0 & + & 0.03 & 1 \\
\hline Feldmannia globifera & 0 & + & + & 0.07 & 2 \\
\hline Colpomenia peregrina & + & + & + & 0.10 & 3 \\
\hline
\end{tabular}




\begin{tabular}{|c|c|c|c|c|c|}
\hline Statement number & 1 & 2 & 3 & \multirow[t]{5}{*}{ RMG } & \multirow[t]{5}{*}{$\mathrm{Pr}$} \\
\hline Month & Mar. & Nov. & June & & \\
\hline Surface in $\mathrm{cm}^{2}$ & 400 & 400 & 400 & & \\
\hline Cover & $100 \%$ & $90 \%$ & $90 \%$ & & \\
\hline Exposition & $\mathrm{N}$ & NE & NW & & \\
\hline Colpomenia sinuosa & + & + & 0 & 0.07 & 2 \\
\hline Bryopsis hypnoides & 0 & 0 & + & 0.03 & 1 \\
\hline Bryopsis muscosa & 0 & + & 0 & 0.03 & 1 \\
\hline Bryopsis plumosa & + & + & 0 & 0.07 & 2 \\
\hline Bryopsis secunda & + & + & 0 & 0.07 & 2 \\
\hline Caulerpa prolifera & 1 & 1 & 1 & 2.50 & 3 \\
\hline Caulerpa racemosa & 2 & 2 & 1 & 10.83 & 3 \\
\hline Codium bursa & 0 & 1 & 0 & 0.83 & 1 \\
\hline Codium effusum & 1 & 1 & 0 & 1.67 & 2 \\
\hline Codium fragile & 2 & 1 & + & 5.87 & 3 \\
\hline Codium tomentosum & 0 & + & 1 & 0.87 & 2 \\
\hline Chaetomorpha aerea & 1 & 1 & 1 & 2.50 & 3 \\
\hline Chaetomorpha mediterranea & 0 & 1 & 1 & 1.67 & 2 \\
\hline Chaetomorpha linum & 0 & 1 & 1 & 1.67 & 2 \\
\hline Cladophora coelothrix & 0 & 1 & 0 & 0.83 & 1 \\
\hline Cladophora laetevirens & 1 & 0 & 0 & 0.83 & 1 \\
\hline Cladophora prolifera & 1 & 1 & 1 & 2.50 & 3 \\
\hline Cladophora coelothrix & + & + & + & 0.10 & 3 \\
\hline Cladophora rupestris & 0 & 0 & 1 & 0.83 & 1 \\
\hline Bldingia marginata & + & + & + & 0.10 & 3 \\
\hline Ulva compressa & 1 & 1 & 1 & 2.50 & 3 \\
\hline Ulva intestinalis & 2 & 1 & 1 & 6.67 & 3 \\
\hline Ulva linza & + & + & + & 0.10 & 3 \\
\hline Ulva prolifera & + & + & + & 0.10 & 3 \\
\hline Ulva clathrata & 0 & 0 & + & 0.03 & 1 \\
\hline Ulva fasciata & + & + & + & 0.10 & 3 \\
\hline Ulva lactuca & 1 & 1 & 1 & 2.50 & 3 \\
\hline Ulva rigida & 2 & 1 & 1 & 6.67 & 3 \\
\hline Number of species per statement & 34 & 49 & 46 & 101.79 & \\
\hline Average number of species per statement & \multicolumn{3}{|l|}{43} & \multirow{3}{*}{\multicolumn{2}{|c|}{ Pr: the presence of the species }} \\
\hline $\mathrm{R} / \mathrm{P}$ per statement & 3.5 & 4.4 & 4.2 & & \\
\hline $\mathrm{R} / \mathrm{P}$ average & \multicolumn{3}{|l|}{4.03} & & \\
\hline
\end{tabular}




\begin{tabular}{|l|l|l|l|l|l|}
\hline Statement number & 1 & 2 & 3 & RMG & Pr \\
\hline Month & Mar. & Nov. & June & \\
\hline Surface in $\mathrm{cm}^{2}$ & 400 & 400 & 400 & & \\
\hline Cover & $100 \%$ & $90 \%$ & $90 \%$ & & \\
\hline Exposition & $\mathrm{N}$ & $\mathrm{NE}$ & $\mathrm{NW}$ & \\
\hline Diversity ndex H' & 4.49 & & & & \\
\hline Evenness E & 0.77 & & & \\
\hline
\end{tabular}

Table 2.

Analytical parameters at the Salamandre Station.

\begin{tabular}{|l|l|l|l|l|}
\hline & IRMG & Dri & Q & DQ \\
\hline Chlorophyceae & $53.91 \%$ & $52.96 \%$ & 19.66 & $46.08 \%$ \\
\hline Phaeophyceae & $2.85 \%$ & $2.79 \%$ & 4 & $9.37 \%$ \\
\hline Rhodophyceae & $45.11 \%$ & $44.31 \%$ & 19 & $44.53 \%$ \\
\hline Total & $101.87 \%$ & & & \\
\hline
\end{tabular}

Table 3.

Algal settlement sheet: Small Port Station.

\begin{tabular}{|c|c|c|c|c|c|c|c|c|c|c|c|}
\hline Statement number & 1 & 2 & 3 & RMG & $\mathrm{Pr}$ & Statement number & 1 & 2 & 3 & RMG & Pr \\
\hline Month & Mar. & Nov. & June & & & Date & Mar. & Nov. & June & & \\
\hline Surface in $\mathrm{cm}^{2}$ & 400 & 400 & 400 & & & Surface in $\mathrm{cm}^{2}$ & 400 & 400 & 400 & & \\
\hline Cover & $100 \%$ & $90 \%$ & $90 \%$ & & & Cover & $100 \%$ & $90 \%$ & $90 \%$ & & \\
\hline Exposition & $\mathrm{N}$ & NE & NW & & & Exposition & $\mathrm{N}$ & NE & NW & & \\
\hline $\begin{array}{l}\text { Porphyra } \\
\text { umbilicalis }\end{array}$ & + & 0 & 0 & 0.03 & 1 & Cladosiphon mediterraneu & + & 0 & 0 & 0.03 & 1 \\
\hline $\begin{array}{l}\text { Pyropia } \\
\text { leucosticta }\end{array}$ & + & 0 & + & 0.07 & 2 & Myriactula gracilariae & 0 & 0 & + & 0.03 & 1 \\
\hline $\begin{array}{l}\text { Sahlingia } \\
\text { subintegra }\end{array}$ & + & + & 0 & 0.07 & 2 & Myriactula rigida & 0 & 0 & + & 0.03 & 1 \\
\hline $\begin{array}{l}\text { Antithamnion } \\
\text { amphigeneum A. } \\
\text { J. K. Millar }\end{array}$ & 0 & 0 & + & 0.03 & 1 & Myriactula rivulariae & 0 & 0 & + & 0.03 & 1 \\
\hline $\begin{array}{l}\text { Asparagopsis } \\
\text { armata }\end{array}$ & 2 & 1 & 0 & 5.83 & 2 & $\begin{array}{l}\text { Ectocarpusfas ciculatus var. } \\
\text { fasciculatus }\end{array}$ & + & 0 & + & 0.07 & 2 \\
\hline $\begin{array}{l}\text { Falkenbergia } \\
\text { rufolanosa }\end{array}$ & 1 & 1 & 0 & 1.67 & 2 & Ectocarpus commensalis & 0 & 0 & + & 0.03 & 1 \\
\hline Anotrichium tenue & + & + & 0 & 0.07 & 2 & Ectocarpus siliculosus & + & 0 & 0 & 0.03 & 1 \\
\hline $\begin{array}{l}\text { Centrocera } \\
\text { clavulatum }\end{array}$ & + & 0 & + & 0.07 & 2 & Feldmannia globifera & + & + & 0 & 0.07 & 2 \\
\hline
\end{tabular}




\begin{tabular}{|c|c|c|c|c|c|c|c|c|c|c|c|}
\hline Statement number & 1 & 2 & 3 & \multirow[t]{5}{*}{ RMG } & \multirow[t]{5}{*}{$\mathrm{Pr}$} & Statement number & 1 & 2 & 3 & \multirow[t]{5}{*}{ RMG } & \multirow[t]{5}{*}{$\mathrm{Pr}$} \\
\hline Month & Mar. & Nov. & June & & & Date & Mar. & Nov. & June & & \\
\hline Surface in $\mathrm{cm}^{2}$ & 400 & 400 & 400 & & & Surface in $\mathrm{cm}^{2}$ & 400 & 400 & 400 & & \\
\hline Cover & $100 \%$ & $90 \%$ & $90 \%$ & & & Cover & $100 \%$ & $90 \%$ & $90 \%$ & & \\
\hline Exposition & $\mathrm{N}$ & $\mathrm{NE}$ & NW & & & Exposition & $\mathrm{N}$ & NE & NW & & \\
\hline $\begin{array}{l}\text { Corallophila } \\
\text { cinnabarina }\end{array}$ & + & + & + & 0.10 & 3 & Feldmannia simplex & + & + & + & 0.10 & 3 \\
\hline $\begin{array}{l}\text { Ceramium } \\
\text { diaphanum }\end{array}$ & 2 & 1 & + & 5.87 & 3 & Feldmannia mitchelliae & 1 & + & 0 & 0.87 & 2 \\
\hline $\begin{array}{l}\text { Spyridia } \\
\text { filamentosa }\end{array}$ & 0 & + & + & 0.07 & 2 & Hincksia sandriana & 1 & + & 0 & 0.87 & 2 \\
\hline Dasya rigidula & + & + & 0 & 0.07 & 2 & Ralfsia verrucosa & 1 & 1 & 0 & 1.67 & 2 \\
\hline Taenio mananum & + & + & 0 & 0.07 & 2 & Cystoseira algeriensis & 1 & + & 1 & 1.70 & 3 \\
\hline $\begin{array}{l}\text { Chondria } \\
\text { coerulescens }\end{array}$ & + & + & + & 0.10 & 3 & Cystoseira barbata & 1 & + & + & 0.90 & 3 \\
\hline $\begin{array}{l}\text { Chondria } \\
\text { dasyphylla }\end{array}$ & + & + & 0 & 0.07 & 2 & Cystoseira compressa & 1 & + & 1 & 1.70 & 3 \\
\hline Chondria mairei & + & + & + & 0.10 & 3 & $\begin{array}{l}\text { Cystoseira brachycarpa var. } \\
\text { balearica }\end{array}$ & 1 & 1 & 1 & 2.50 & 3 \\
\hline Digenea simplex & 1 & 1 & 0 & 1.67 & 2 & Cystoseira crinita & 0 & 0 & 2 & 5.00 & 1 \\
\hline Halopithys incurva & 1 & 1 & 1 & 2.50 & 3 & Cystoseira sedoides & 1 & 1 & 1 & 2.50 & 3 \\
\hline $\begin{array}{l}\text { Herposiphonia } \\
\text { secunda f. } \\
\text { secunda }\end{array}$ & + & 0 & + & 0.07 & 2 & Cystoseira mediterranea & 1 & + & 1 & 1.70 & 3 \\
\hline $\begin{array}{l}\text { Herposiphonia } \\
\text { secunda f. tenella }\end{array}$ & + & 0 & + & 0.07 & 2 & Cystoseira amentacea var. stricta & 1 & + & 1 & 1.70 & 3 \\
\hline $\begin{array}{l}\text { Laurencia } \\
\text { microcladia }\end{array}$ & 1 & + & 1 & 1.70 & 3 & Cystoseira tamariscifolia & 1 & 1 & 1 & 2.50 & 3 \\
\hline Laurencia obtusa & 0 & 1 & 1 & 1.67 & 2 & Sargassum acinarium & 0 & 0 & 1 & 0.83 & 1 \\
\hline Palisada perforata & + & 1 & 1 & 1.70 & 3 & Sargassum vulgare & 1 & 0 & + & 0.87 & 2 \\
\hline $\begin{array}{l}\text { Lophocladia } \\
\text { lallemandii }\end{array}$ & + & 0 & 0 & 0.03 & 1 & Colpomenia peregrine & 1 & 1 & 1 & 2.50 & 3 \\
\hline $\begin{array}{l}\text { Ellisolandia } \\
\text { elongata }\end{array}$ & 2 & 1 & + & 5.87 & 3 & Colpomenia sinuosa & 1 & 1 & 1 & 2.50 & 3 \\
\hline Corallina officinalis & 1 & 1 & 1 & 2.50 & 3 & Cladostephus spongiousus & 1 & + & 0 & 0.87 & 2 \\
\hline Jania longifurca & 1 & + & + & 0.90 & 3 & Sphacelaria cirrosa & + & 0 & + & 0.07 & 2 \\
\hline Jania rubens & 1 & + & + & 0.90 & 3 & Sphacelaria plumula & + & 0 & + & 0.07 & 2 \\
\hline $\begin{array}{l}\text { Gelidiella } \\
\text { ramellosa }\end{array}$ & + & 0 & 1 & 0.87 & 2 & Halopteris scoparia & 0 & 1 & 1 & 1.67 & 2 \\
\hline Gelidium crinale & + & 0 & 1 & 0.87 & 2 & Bryopsis duplex & + & + & 0 & 0.07 & 2 \\
\hline $\begin{array}{l}\text { Gelidium } \\
\text { spinosum }\end{array}$ & 0 & 0 & 1 & 0.83 & 1 & Bryopsis hypnoides & + & + & 0 & 0.07 & 2 \\
\hline Gelidium corneum & + & 0 & 0 & 0.03 & 1 & Bryopsis muscosa & + & 0 & 0 & 0.03 & 1 \\
\hline $\begin{array}{l}\text { Pterocladiella } \\
\text { capillacea }\end{array}$ & + & 0 & 0 & 0.03 & 1 & Bryopsis plumosa & + & 0 & 0 & 0.03 & 1 \\
\hline
\end{tabular}




\begin{tabular}{|c|c|c|c|c|c|c|c|c|c|c|c|}
\hline Statement number & 1 & 2 & 3 & RMG & $\operatorname{Pr}$ & Statement number & 1 & 2 & 3 & RMG & $\operatorname{Pr}$ \\
\hline Month & Mar. & Nov. & June & & & Date & Mar. & Nov. & June & & \\
\hline Surface in $\mathrm{cm}^{2}$ & 400 & 400 & 400 & & & Surface in $\mathrm{cm}^{2}$ & 400 & 400 & 400 & & \\
\hline Cover & $100 \%$ & $90 \%$ & $90 \%$ & & & Cover & $100 \%$ & $90 \%$ & $90 \%$ & & \\
\hline Exposition & $\mathrm{N}$ & NE & NW & & & Exposition & $\mathrm{N}$ & NE & NW & & \\
\hline $\begin{array}{l}\text { Chondracanthus } \\
\text { acicularis }\end{array}$ & 1 & 1 & + & 1.70 & 3 & Bryopsis secunda & + & + & 0 & 0.07 & 2 \\
\hline $\begin{array}{l}\text { Chondracanthus } \\
\text { teedei }\end{array}$ & 0 & 1 & + & 0.87 & 2 & Caulerpa prolifera & 1 & + & 1 & 1.70 & 3 \\
\hline $\begin{array}{l}\text { Rissoella } \\
\text { verruculosa }\end{array}$ & + & + & 0 & 0.07 & 2 & Codium bursa & 0 & 1 & 1 & 1.67 & 2 \\
\hline $\begin{array}{l}\text { Gracilaria bursa- } \\
\text { pasoris }\end{array}$ & 1 & + & + & 0.90 & 3 & Codium effusum & 1 & + & 1 & 1.70 & 3 \\
\hline $\begin{array}{l}\text { Gracilariopsis } \\
\text { longissima }\end{array}$ & + & + & 0 & 0.07 & 2 & Codium fragile & 1 & 0 & 1 & 1.67 & 2 \\
\hline $\begin{array}{l}\text { Hypnea } \\
\text { musciformis }\end{array}$ & 2 & 2 & 1 & 10.83 & 3 & Codium tomentosum & 0 & 1 & 1 & 1.67 & 2 \\
\hline Grateloupia filicina & + & 0 & + & 0.07 & 2 & Chaetomorpha aerea & 1 & 1 & 2 & 6.67 & 3 \\
\hline $\begin{array}{l}\text { Peyssonnelia } \\
\text { polymorpha }\end{array}$ & + & 0 & + & 0.07 & 2 & Chaetomorpha mediterranea & 1 & 1 & 1 & 2.50 & 3 \\
\hline $\begin{array}{l}\text { Peyssonnelia } \\
\text { rubra }\end{array}$ & 0 & 0 & + & 0.03 & 1 & Cladophora coelothrix & 0 & 1 & + & 0.87 & 2 \\
\hline $\begin{array}{l}\text { Peyssonnelia } \\
\text { squamaria }\end{array}$ & + & 0 & 0 & 0.03 & 1 & Cladophora laetevirens & 1 & 1 & 1 & 2.50 & 3 \\
\hline $\begin{array}{l}\text { Sphaerococcus } \\
\text { coronopifolius }\end{array}$ & 0 & + & 0 & 0.03 & 1 & Cladophora prolifera & + & 0 & 0 & 0.03 & 1 \\
\hline $\begin{array}{l}\text { Nemalion } \\
\text { helminthoides }\end{array}$ & + & + & 0 & 0.07 & 2 & Acetabularia acetabulum & + & 0 & 0 & 0.03 & 1 \\
\hline Liagora viscida & + & 0 & 1 & 0.87 & 2 & Bldingia marginata & + & + & + & 0.10 & 3 \\
\hline $\begin{array}{l}\text { Gastroclonium } \\
\text { clavatum }\end{array}$ & + & 0 & 0 & 0.03 & 1 & Ulva compressa & 1 & 1 & 1 & 2.50 & 3 \\
\hline $\begin{array}{l}\text { Irvinea } \\
\text { boergesenii }\end{array}$ & + & 0 & 0 & 0.03 & 1 & Ulva intestinalis & + & 0 & 0 & 0.03 & 1 \\
\hline Cutleria adspersa & 0 & 0 & + & 0.03 & 1 & Ulva linza & + & + & 0 & 0.07 & 2 \\
\hline $\begin{array}{l}\text { Dictyota } \\
\text { dichotoma }\end{array}$ & 1 & 1 & 0 & 1.67 & 2 & Ulva prolifera & + & 1 & 1 & 1.70 & 3 \\
\hline Dictyota fasciola & 1 & + & 0 & 0.87 & 2 & Ulva clathrata & 2 & 1 & 1 & 6.67 & 3 \\
\hline $\begin{array}{l}\text { Dictyopteris } \\
\text { polypodioides }\end{array}$ & 2 & 0 & 1 & 5.83 & 2 & Ulva fasciata & + & + & + & 0.10 & 3 \\
\hline Dictyota spiralis & 0 & + & 1 & 0.87 & 2 & Ulva lactuca & 1 & 1 & 1 & 2.50 & 3 \\
\hline Padina pavonica & 0 & 0 & + & 0.03 & 1 & Ulva rigida & 2 & 1 & 1 & 6.67 & 3 \\
\hline \multirow[t]{2}{*}{$\begin{array}{l}\text { Asperococcus } \\
\text { bullosus }\end{array}$} & + & 0 & + & 0.07 & 2 & & & & & & \\
\hline & & & & & & Number of species per $\mathrm{s}$ & 89 & 68 & 72 & 130.4 & \\
\hline
\end{tabular}




\begin{tabular}{|c|c|c|c|c|c|c|c|c|c|c|c|}
\hline Statement number & 1 & 2 & 3 & \multirow[t]{5}{*}{ RMG } & \multirow[t]{5}{*}{$\mathrm{Pr}$} & Statement number & 1 & 2 & 3 & \multirow[t]{5}{*}{ RMG } & $\mathrm{Pr}$ \\
\hline Month & Mar. & Nov. & June & & & Date & Mar. & Nov. & June & & \\
\hline Surface in $\mathrm{cm}^{2}$ & 400 & 400 & 400 & & & Surface in $\mathrm{cm}^{2}$ & 400 & 400 & 400 & & \\
\hline Cover & $100 \%$ & $90 \%$ & $90 \%$ & & & Cover & $100 \%$ & $90 \%$ & $90 \%$ & & \\
\hline \multirow[t]{6}{*}{ Exposition } & $\mathrm{N}$ & NE & NW & & & Exposition & $\mathrm{N}$ & NE & NW & & \\
\hline & & & & & & $\begin{array}{l}\text { Average number of species } \\
\text { per statement }\end{array}$ & \multicolumn{3}{|l|}{76.33} & \multirow{3}{*}{\multicolumn{2}{|c|}{$\begin{array}{l}\text { Pr: the } \\
\text { presence } \\
\text { of the } \\
\text { species }\end{array}$}} \\
\hline & & & & & & $\mathrm{R} / \mathrm{P}$ per statement & 1.61 & 1.45 & 1.07 & & \\
\hline & & & & & & R/P Average & \multicolumn{3}{|l|}{1.37} & & \\
\hline & & & & & & Diversity index H' & \multicolumn{3}{|l|}{5.56} & & \\
\hline & & & & & & Evenness $\mathrm{E}$ & \multicolumn{3}{|l|}{0.82} & & \\
\hline
\end{tabular}

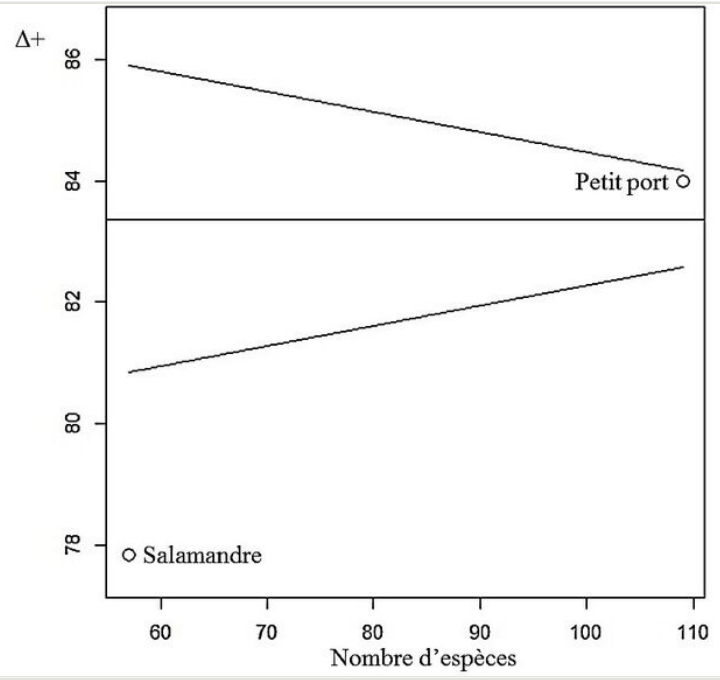

Figure 3. doi

Funnel test.

Table 4.

Analytical parameters at the Small Port Station.

\begin{tabular}{|l|l|l|l|l|}
\hline & LRMG & Dri & Q & DQ \\
\hline Chlorophyceae & $31.69 \%$ & $24.30 \%$ & 19 & $25.11 \%$ \\
\hline Phaeophyceae & $42.61 \%$ & $32.67 \%$ & 24 & $31.72 \%$ \\
\hline Rhodophyceae & $56.10 \%$ & $43.02 \%$ & 32.66 & $43.17 \%$ \\
\hline Total & $130.40 \%$ & & & \\
\hline
\end{tabular}




\section{Table 5.}

Taxonomic diversity indices.

\begin{tabular}{|l|l|l|l|l|}
\hline Region & S & delta & delta* & delta+ \\
\hline Small port & 109 & 79.98 & 81.80 & 83.99 \\
\hline Salamandre & 57 & 72.163 & 76.26 & 77.84 \\
\hline Expected & & 160.61 & 78.59 & 83.37 \\
\hline
\end{tabular}

The results shown in Table 1 and Table 3, with illustrations and descriptions of sample specimens for the two observation stations are contained in a doctoral thesis (Bachir Bouiadjra 2012).

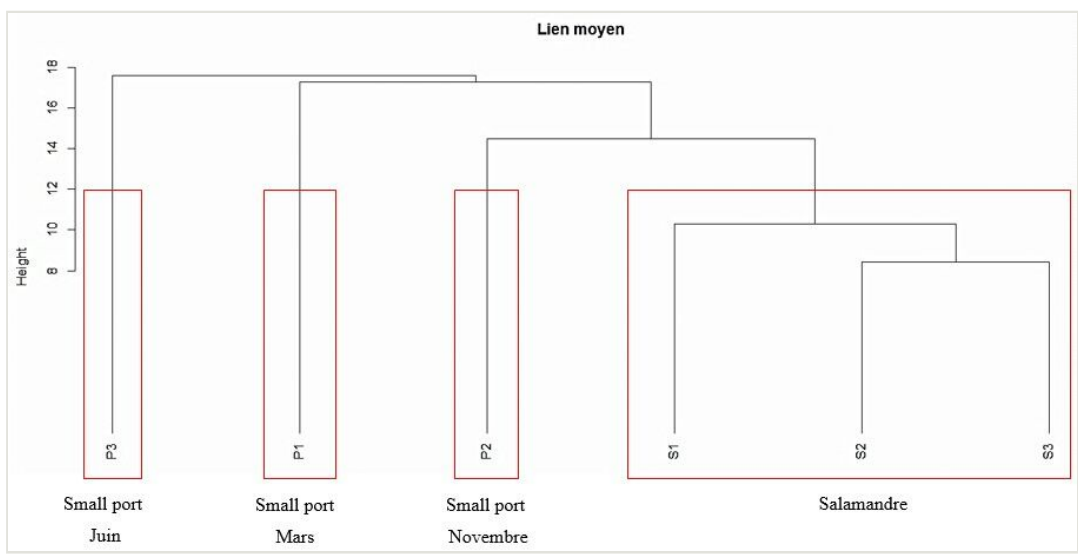

Figure 4. doi

Classification of phytosociological surveys carried out at the Small Port and the Salamandre Station by the average link.

\section{Discussion}

\section{Salamandre Station}

The reading of the sheet on the algal settlement of the Salamandre site (Table 1) and the result of the structural parameters shown in Table 2, indicate a quantitative and qualitative dominance, respectively of $52 \%$ and $46 \%$ with an overall average recovery exceeding $50 \%$ of the species belonging to Chlorophyceae. Within this systematic unit, we note an overall average recovery rate of five invasive taxa, C. cylindracea, C. fragile, A. armata, F. rufolanosa L. lallemandii, which is of the order of $24.5 \%, 10.5 \%$ of which is reserved for the C. cylindracea, which is progressing in some surveys by joining its small colonies (Figs 5 , 6) to which are added the eutrophication indicator species of the environment and nitrophilic strongly accumulating nitrogen are represented by the order of Ulvales in the number of 8 species (Table 1) with a global average cover rate of $18 \%$ on a total global 
cover of $50 \%$ of Chlorophyceae in the Salamandre Station. This high presence rate is an indicator of a polluted environment which is confirmed by domestic and urban discharges (Khiari et al. 2017, Damak et al. 2019) duly observed at Salamandre Station, in addition the presence rate of the invasive species $C$. cylindracea amount to $10.83 \%$ (Table 1) which confirms an increasingly advanced colonisation.

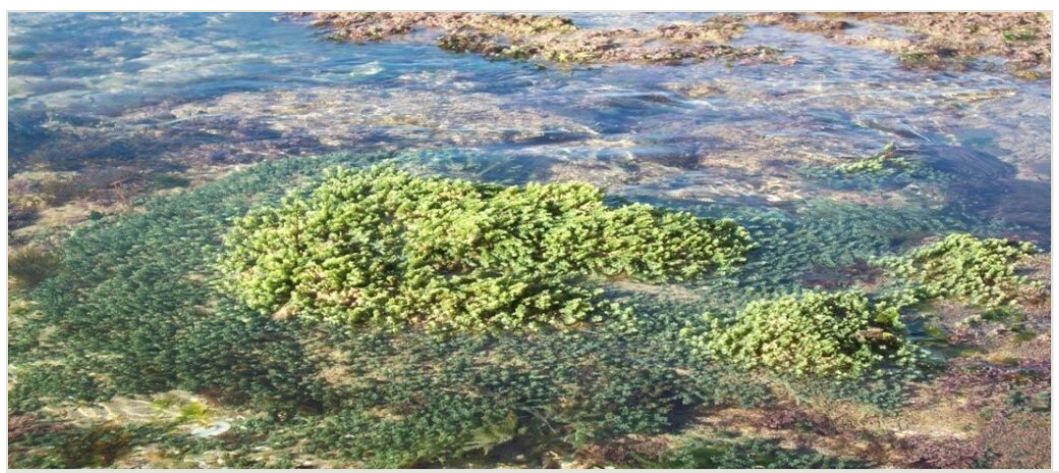

Figure 5. doi

Central colonies of $C$. cylindracea at the Salamandre Station.

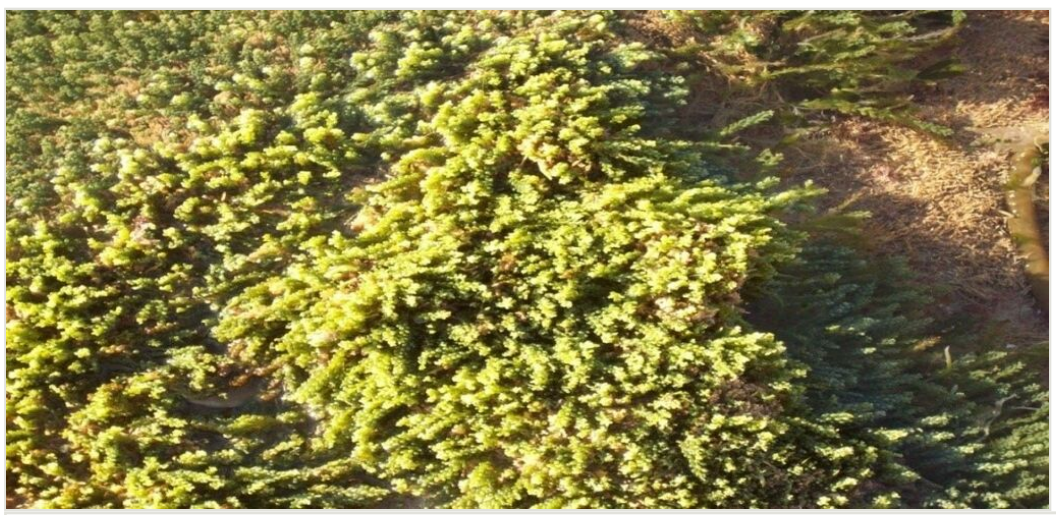

Figure 6. doi

Dense colonies of Caulerpa cylindracea in the heavily affected station of Salamandre.

The algal population of Phaeophyceae occupies a negligible average cover rate of $2.85 \%$ at the Salamandre Station and reveals the total absence of macrophyte species sensitive to pollution by detergents and water turbidity such as cystoseires (Cystoseira stricta, C. algeriensis, C. crinita ... etc) (Gamulin-Brida et al. 1967, Bellan-Santini 1966, Verlaque and Fritayre 1994a) which confirms that this site is strongly impacted by C. cylindracea and disturbed, given the strong presence of the invasive species On the other hand the site of the small harbor displays a rate of recovery of Phaeophyceae macrophytes of $42.61 \%$ (Table 4) with a presence of nine species of Cystoseires suggesting a good water quality and an average algal specific richness of 76 species (Table 3) per survey contrary to the 
previous site, which displays an average number of species of only 43 per survey (Table 1).

As for Rhodophyceae the overall cover rate is respectively $45.11 \%$ and $56.10 \%$ at the Salamandre and small port Station with a qualitative dominance of $44 \%$ and $43 \%$ (Tables $2,4)$ which suggests protection and valorisation of some taxa rich in mineral elements and chemical components (Sambusiti et al. 2015, Cardoso et al. 2014, Shobana et al. 2017) see Ellisolandia elongata, Hypnea musciformis, Gelidium crinale, Gelidium spinosum, Gelidium corneum, Jania longifurca, Jania rubens and Gracilaria bursa-pasoris.

This is easily verified in the number of species per phytosociological survey, which is much lower than those reported in the Small Port site and the reduction in the Diversity and Evenness Indices, respectively by 4.49 and 0.77 at the Salamandre Station, in relation to the results obtained at the Small Port. In this station invaded by C. cylindracea, a native species, Caulerpa prolifera (Forsskal) Lamouroux are recorded in surveys, with a low recovery rate of $2.5 \%$ compared to that of the invasive taxon $(10.5 \%)$ suggesting a tendency for $C$. prolifera to be replaced by the invasive species (Piazzi and Ceccherelli 2006). Over time, according to Ceccherelli et al. 2001, Jaubert et al. 2003), the homogenisation of the underwater landscape observed in some Mediterranean regions heavily affected by the invasive species has resulted. It is important to remember that for the four other invasive species of Rhodhophycea inventoried and subservient to the photophilic infralittoral of hard substrates, seem to find favorable conditions for their development, without any threat to the ecosystem. Nevertheless, the Bonnemaisonncea of the genus Asparagpsis found in Salamandre, has major assets its avoidance by herbivorous fish because secreting secondary metabolites and its ability to multiply vegetatively and by apomeiotic spores (Feldmann 1954) hence its expansion to the site of Salamandre with a recovery rate of $1 \%$, thus forming a settlement in Asparagpsis.

In addition, the beach of Salamandre with coastal developments operating in recent years is a vulnerable site to closely monitor to avoid erosion of its algal richness due to an expansion of invasive taxon C. cylindracea.

\section{Small Port Station}

It is a site rich in species, 109 taxa listed (Table 3), $80 \%$ of the total number recorded on the coast of Mostaganem (Bachir Bouiadjra 2012). The recovery rate of the entire algal flora exceeds $100 \%$, with a Diversity Index $\left(\mathrm{H}^{\prime}\right)$ of 5.56 which is higher than that of the previous station (Salamandre), as well as that of Bordj El kifan (1.62) east of Algiers reported by Serridi (2003). The Evenness Index is about 0.82 , which suggests, according to Daget (1979), a balanced algal stand.

The overall average overlap among Rhodophyceae is clearly dominant at $56 \%$, the same observation is made for their quantitative and qualitative dominance estimated at $43 \%$, followed by the Phaeophyceae group with an RMG of $42 \%$ and dominance (Dri) and (DQ) of $32 \%$ and finally the Chlorophyceae group with an RMG of $31 \%$ (Table 4). This represents a well-structured settlement with species constituting the crusty, grassy, erect 
and epiphytic strata according to the algal settlement sheet (Table 3). Note the absence of the invasive taxon $C$. cylindracea however, the fishing port adjacent to the observation sites, will constitute a potential vector for caulerpe transmission in the area. Hence the need for increased vigilance and follow-up with awareness campaigns among fishing professionals to avoid contamination of the site.

\section{Taxonomic Diversity Indices}

Analysis of the different taxonomic Diversity Indices (Table 5) indicates a higher taxonomic diversity at the Small Port than at the Salamandre Station. This means that the species recorded at the Small Port are more taxonomically distant than those found in Salamandre. In other words, the average taxonomic distance between each pair of randomly selected individuals is greater at the Small Port than at Salamandre.

The Funnel Test (Fig. 3) based on the values of $\Delta^{+}$obtained for each Station, in comparison with the mean expected value in the study area, shows that the Salamandre Station is outside the $95 \%$ confidence interval of $\Delta+\mathrm{m}$, indicating a low taxonomic diversity compared to that expected in the area. This suggests that this site is subject to anthropogenic disturbances, influencing the taxonomic composition of the settlement by generating a poor assemblage of species, in addition to the presence of invasive species in this plant, associated with untreated wastewater discharges, confirm this reduction in algal diversity (Verlaque and Fritayre 1994b, Ceccherelli et al. 2001, Piazzi et al. 2001, Piazzi and Cinelli 2003, Piazzi and Ceccherelli 2006).

\section{Hierarchical Ascendant Clustering}

Our study was confirmed by a multidimensional analysis which is the Hierarchical Ascendant Clustering (HAC). The latter (Fig. 4) shows a clear separation between the two sites considered by indicating four distinct groups of phytosociological surveys. The first two, represented by the summer and spring surveys at the Small Port Station, are the most dissimilar of the other groups and represent the greatest distance. The two surveys, therefore, represent the most species-rich removal recorded, indicating 89 and 72 species recorded respectively during the spring and summer seasons at the Small Port. However, we note a decrease in the number of species in the surveys conducted during the winter season at the same site with 68 species recorded, which results in a smaller distance in the classification tree for this survey. The last group consists of the surveys carried out in the Salamandre Station and represents the smallest distance in the dendrogram due to the low number of species recorded due to the dominance of the settlementby species belonging to Chlorophytes, neutrophils associated with five other invasive species including C. cylindracea, C. fragile, A. armata, F. rufolanosa and L. lallemandii, which testifies to the disturbance and degradation of the environment in the Salamandre Station. 


\section{Conclusions}

The invasive alga Caulerpa cylindracea Sonder, 1845 tends to colonise disturbed ecosystemseflecting a reduction in indigenous algal diversity (Ceccherelli et al. 2001, Piazzi et al. 2001, Piazzi and Cinelli 2003, Piazzi and Ceccherelli 2006). This is confirmed in the heavily colonised observation site of Salamandre Beach, through the statistical analysis tools used to see the parameters of abundance recovery, qualitative and quantitative dominance of algal groups, Equitability Index and taxonomic diversity and where there is a drastic depletion of the invaded algal community, represented by a limited number of Macrophyte algae accompanying the invasive taxon in phytosociological surveys and a low Shannon-Weaver Diversity Index $(\mathrm{H})$ of 4.49 . On the other hand, the Station of the Small Port not affected by $C$. cylindracea hosts a population exceeding in some surveys about twenty taxa or the Diversity Index is 5.56 suggesting a well-structured and stable algal population. The number of macroalgal species accompanying the invasive species has dropped by $52 \%$ in Salamandre. Verlaque and Fritayre (1994b) attribute this decrease to a rate ranging from 25 to $55 \%$. This is consistent with our results. Furthermore, the analysis of the floral procession mentioned in the surveys of the Salamandre Station indicates a decrease in the cover of erect shrub algae $(-70$ to $-80 \%)$ and epiphytic and filamentous algae in the autumn season, while encrusting algae of the genus Corallina dominate and best resist the occupation of the ecological niche by the invasive species $C$. cylindracea. This study provides an inventory of the algal population associated with the recentlyintroduced $C$. cylindracea at the Mostaganem coast, which needs to be monitored at different levels of the affected coastline in order to effectively limit its expansion to the detriment of existing algal diversity.

\section{Acknowledgements}

The authors would like to thank the Directorate General of Scientific Research and Technological Development for its accompaniment of the Projects for University Research and Formation.

\section{References}

- Bachir Bouiadjra B (2012) Étude de la flore algale benthique et les impacts de ses espèces invasives devant la côte Mostaganemoise. Thèse de doctorat. Université Abdelhamid Ibn Badis de Mostaganem, Algérie, 143 pp. URL: http://e-biblio.univmosta.dz/handle/123456789/1092

- Ballesteros E, Toras X, Pinedo S, Garcia M, Mangialajo L, De Tores M (2007) A new methodology based on littoral community cartography dominated by macroalgae for the implementation of the European Water framework directive. Marine Pollution Bulletin 55 (2007): 172-180. https://doi.org/10.1016/j.marpolbul.2006.08.038

- Belhissoun S (1995) Contribution à l'étude Phyto-écologique du Phytobenthos Marin de la région de Rabat- Mohammedia (Atlantique Marocain). Thèse du diplme d'études 
supérieures de 3ème Cycle. Université Mohammed V. Faculté des Sciences. Rabat, Maroc.

- Bellan-Santini D (1966) influences des eaux usées sur la faune et la flore marine benthique dans la région Marseillaise. Techniques et sciences municipales 61 (7): 285-292.

- $\quad$ Bliding C (1968) A critical survey of European taxa in Ulvales, II: Ulva, Ulvaria, Monostroma, Kormania. Botaniska Notiser 121: 535-629.

- Boudouresque CF, Boudouresque E (1969) Contribution à la flore des Algues de l'Algérie. Bulletin du Musée d'histoire naturelle de Marseille. Fr 29: 19-36.

- Boudouresque CF (1971a) Méthodes d'étude qualitative et quantitative du benthos (en particulier le phytobenthos). Téthys, Fr 3 (1): 79-104.

- Boudouresque CF (1971b) Recherche de bionomie analytique, structurale et exprimentale sur les peuplements benthiques sciaphiles de Méditerranée occidentale (fraction algale). La sous-stratesciaphile des peuplements de grande Cystoseira de mode battu. Bulletin du Musée d'Histoire Naturelle de Marseille, Fr 31: 141-151.

- $\quad$ Boudouresque CF, Cinelli F (1971) The algae population of superficial biotopes sciaphiles of the kind found in the sland of Ischia (Naples Gulf, Italy). Pubblicazioni della Stazione Zoologica Napoli, Italy 39: 1-43.

- Bouroche JM, Saporta G (2005) L'analyse des données. 9ème edition. Presses Universitaires de France, Paris, 127 pp.

- Bright C (1998) Life out of bounds. Bioinvasion in a Borderless Word. Worlwath Institute: Norton Publication.

- $\quad$ Brunel S, Fernandez-Galiano E, Genovesi P, Heywood VH, Kueffer C, Richardson DM (2013) Invasive alien species: A growing but neglected reat? In late lessons from early warning: Science, precaution, innovation. essons for preventing harm. EEA Report 1/2013, Copenhagen518-540.

- Cardoso S, Carvalho L, Silva P, Rodrigues M, Pereira O, Pereira L (2014) Bioproducts from Seaweeds: A Review with Special Focus on the Iberian Peninsula. Current Organic Chemistry 18 (7): 896-917. https://doi.org/10.2174/1385272818071405 15154116

- Carlton J, (1985) transoceanic and anter oceanic dispersal of coasal marine organisms: the biology of ballast water. Oceanography and arine iology 3: 265-273.

- Carrillo Marta Sanson JA (1999) Algas, Hongros y fanerogamas marinas de Las Islas Canarias. Clave analitica. Service de publicaciones. Universidade de la Laguna, 143 pp.

- Ceccherelli G, Piazzi I, Cinelli F, Gravez V, Ruitton S, Boudouresque CF, Le Direac'h L, Meinesz A, Scabbia G, Verlaque M (2001) The development of Caulerpa racemosa at the margin of posidonia oceanica. Fourth international workshop on Caulerpa taxifolia edition, GIS Posidonie Publication.376-384.

- $\quad$ Clarke KR, Warwick RM (1998) A taxonomic distinctness index and its statistical properties. Journal of Applied Ecology 35: 523-531. https://doi.org/10.1046/j. 1365-2664.1998.3540523.x

- $\quad$ Clarke KR, Warwick RM (1999) The taxonomic distinctness measure of biodiversity: weighting of step lengths between hierarchical levels. Marine Ecology Progress Series 184: 21-29. https://doi.org/10.3354/meps184021 
- Coppejans E (1983) Iconographie d'Algues Méditerranéennes. Chlorophyta, Phaeophyta, Rhodophyta. Bibliothecaphycologica, Herausgegebenvon.

J. Cramer, 317 pp.

- Cormaci M, Lanfranco E, Borg JA, Buttigieg S, Furnari G, Micallef SA, Mifsud C, Pizzuto F, Scammacca B, Serio D (1997) Contribution to the Knowledge of benthic marine algae on rocky substrata of the Maltese Islands (Mediterranean Sea). Botanica Marina. 40: 203-215.

- Daget J (1979) Les modèles mathématiques en écologie. 8ème ed. Collection d'écologie. Masson, $172 \mathrm{pp}$.

- $\quad$ Dagnelie $P$ (1986) Analyse statistique à plusieurs variables. 3 ième réimpression. Les presses agronomiques de Gembloux, Gembloux, $362 \mathrm{pp}$.

- Damak M, Frontalini F, Elleuch B, Kallel M (2019) Benthic foraminiferal assemblages as pollution proxies along the coastal fringe of the Monastir Bay (Tunisia). Journal of African Earth Sciences 150: 379-388. https://doi.org/10.1016/j.jafrearsci.2018.11.013

- Feldmann J (1931) Contribution à la flore Algologique Marine de l'Algérie. Les Algues de Cherchell. Bulletin de la Société d'Histoire Naturelle de l'Afrique du Nord Alger 22: 229-254.

- Feldmann J (1933) Contribution à la flore Algologique Marine de l'Algérie. Bulletin de la Société d'Histoire Naturelle de l'Afrique du Nord Alger 24 (Fascicule 2): 360-366.

- Feldmann J (1954) Inventaire de la flore marine de Roscoff (algues, champignons, lichens et spermatophytes). Travaux de la Station Biologique de Roscoff. Fr 6 (suppl.): 1-252.

- $\quad$ Fischer W, Schneider M, Bauchot ML (1987) Méditerranée et Mer Noire, Zone de pêche 37 Révision 1, volume 1, Végétaux et Invertébrés. Fiches d'identification, 760 pp.

- Gamulin-Brida H, Giaccone G, Golubić S (1967) Contribution aux études des biocoenoses subtidales. Helgoländer Wissenschaftliche Meeresuntersuchungen 15: 429-444. https://doi.org/10.1007/bf01618639

- Gayral P (1958) Algues de la côte Atlantique Marocaine. La nature au Maroc II, Casablanca, $523 \mathrm{pp}$.

- Gayral P (1966) Les Algues des Côtes Françaises (Manche et Atlantique). Notions Fondamentales sur l'écologie, la biologie et la systématique des Algues Marines. Edition Doin- Deren \& Cie, 632 pp.

- Giakoumi S (2014) Distribution patterns of the invasive herbivore Siganus luridus (Ruppell, 1829) and its relation to native benthic communities in the Central Aegean Sea, Northeastern Mediterranean. Marine Ecology 35 (12059): 96-105. https://doi.org/ 10.1111/maec.12059

- $\quad$ Giakoumi S, Katsanevakis S, Albano PG, Azzurro E, Cardoso AC, Cebrian E (2019) Management priorities for marine invasive species. Science of the Total Environment 688: 976-982. https://doi.org/10.1016/j.scitotenv.2019.06.282

- Gounout M (1969) Méthodes d'étude quantitative de la végétation. Masson édit, Paris, $320 \mathrm{pp}$.

- $\quad$ Gribben P, Thomas T, Pusceddu A, Bonechi L, Bianchelli S, Buschi E, Nielsen S, Ravaglioli C, Bulleri F (2018) Below-ground processes control the success of an invasive seaweed. Journal of Ecology 106 (5): 2082-2095. https://doi.org/ 10.1111/1365-2745.12966

- Guiry MD, Guiry GM (2020) Algae-Base. World-wide electronic publication, National University of Ireland, Galway (taxonomic information republished from Algae-Base with 
permission of M.D Guiry). Accessed through: World Register of Marine Species at: http://www. marinespecies.org/aphia. php?p=taxdetails\&id=382517 on 2020-03-28.

- Hamel G (1931) Chlorophycées des côtes Françaises. Revue algologique T. 5-6 33 pp.

- Hamel G (1939) Phaeophycées de France. F. I-V, Paris, 432 pp.

- Herrera RR, Legac DS (2002) Initiation à l'analyse factorielle des données: fondements mathématiques et interprétations. Ellipses, Paris, $315 \mathrm{pp}$.

- Jaubert JM, Chisholm JR, Minghelli-Roman A, Marchioretti M, Morrow JH, Ripley HT (2003) Re-evaluation of the extent of Caulerpa taxifolia development in the northern Mediterranean using airborne spectrographic sensing. Marine Ecology Progress Series 263: 75-82. https://doi.org/10.3354/meps263075

- Khiari N, Atoui A, Khalil N, Charef A, Aleya L (2017) Dynamics of sediments along with their core properties in the Monastir-Bekalta coastline (Tunisia, Central Mediterranean). Journal of African Earth Sciences 134: 320-331. https://doi.org/10.1016/j.jafrearsci. 2017.06.028

- $\quad$ Legendre L, Legendre P (1984) Ecologie numérique. Tome 1. Le traitement multiple des données écologiques: 260 p. Tome 2. La structure des données écologiques: 247 p. 2 ème édition. Collection d'écologie 12. Masson, Paris.

- $\quad$ Legendre P, Legendre L (1998) Numerical Ecology. Elsevier science, Amsterdam, 853 pp. https://doi.org/10.1016/S0167-8892(98)80052-3

- Manconi R, Padiglia A, Padedda BM, Pronzato R (2020) Invasive green algae in a western Mediterranean Marine Protected Area: interaction of photophilous sponges with Caulerpa cylindracea. Journal of the Marine Biological Association of the United Kingdom 100 (3): 361-373. https://doi.org/10.1017/s0025315420000193

- Mannino A, Balistreri P, Deidun A (2017) The marine biodiversity of the Mediterranean Sea in a changing climate: The impact of biological invasions. Mediterranean Identities Environment, Society, Culture https://doi.org/10.5772/intechopen.69214

- Mojetta A, Ghisotti A (1996) Flore et Faune de la Méditerranée. Edition Solar, Paris, 317 pp.

- Montefalcone M, Morri C, Parravicini V, Bianchi CN (2015) A tale of two invaders: divergent spreading kinetics of the alien green algae Caulerpa taxifolia and Caulerpa cylindracea. Biological Invasions 17 (9): 2717-2728. https://doi.org/10.1007/s10530015-0908-1

- $\quad$ Ojaveer H, Galil BS, Carlton JT, Alleway H, Goulletquer P, Lehtiniemi M, et al. (2018) Historical baselines in marine bioinvasions: implications for policy and management. PLOS One 13 (e0202383). https://doi.org/10.1371/journal.pone.0202383.

- Piazzi L, Ceccherelli G, Cinelli F, Gravez V, Ruitton S, Boudouresque CF, Le Direac'h L, Meinesz A, Scabbia G, Verlaque M (2001) Effet de Caulerpa racemosa sur la structure des communautés algales benthiques. Fourth international workshop on Caulerpa taxifolia edition, GIS Posidonie ublication. Fr: 371-37.

- Piazzi L, Cinelli F (2003) Evaluation of benthic macroalgal invasion in a harbour area of the western Mediterranean Sea. European Journal of Phycology 38: 223-231. https://doi.org/10.1080/1364253031000136358

- $\quad$ Piazzi L, Ceccherelli G (2006) Persistence of biological invasion effects: Recovery of macroalgal assemblages after removal of Caulerpa racemosa var. cylindracea. Estuarine Coastal and Shelf Science 68: 455-461. https://doi.org/10.1016/j.ecss. 2006.02.011 
- $\quad$ Piazzi L, Balata D, Bulleri F, Gennaro P, Ceccherelli G (2016) The invasion of Caulerpa cylindracea in the Mediterranean: the known, the unknown and the knowable. Marine Biology 163 (7). https://doi.org/10.1007/s00227-016-2937-4

- Premarathna AD, Sarvananda L, Jayasooriya AP, Amarakoon SA (2019) Review on pathogenic diseases on corals associated risk factors and possible devastations in future in the globe. Journal of Marine Science: Research \& Development 9 (269).

- $\quad$ R Core Team (2014) R: A language and environment for statistical computing. R Foundation for Statistical Computing, Vienna, Austria.

- Sambusiti C, Bellucci M, Zabaniotou A, Beneduce L, Monlau F (2015) Algae as promising feedstocks for fermentative biohydrogen production according to a biorefinery approach: A comprehensive review. Renewable and Sustainable Energy Reviews 44: 20-36. https://doi.org/10.1016/i.rser.2014.12.013

- Scammacca B, Giaccone G, Pizzuto F, Alongi G (1993) La vegetazione marina di substratoduro del l'isola di Lampedusa (Isole Pelagie). Bollettino Accademia Gioenia di Scienze Naturali 26 (341): 85-126.

- Serridi H (2003) Algues Macrophytes, biodiversité Marine et littorale Algérienne. ISBN N9961- 9547-O-X79-94.

- Shobana S, Kumar G, Bakonyi P, Saratale G, Al-Muhtaseb AH, Nemestóthy N, BélafiBakó K, Xia A, Chang J (2017) A review on the biomass pretreatment and inhibitor removal methods as key-steps towards efficient macroalgae-based biohydrogen production. Bioresource Technology 244: 1341-1348. https://doi.org/10.1016/j.biortech. 2017.05.172

- Verlaque M (1990) Végétation Marine de la Corse (Méditerranée) VIII. Documents pour la flore des Algues. Vie et Milieu 40 (1): 79-92.

- Verlaque M, Fritayre P (1994a) Incidence de l'algue introduite Caulerpa taxifolia sur le phytobenthos de Méditerranée occidentale. - 2. Les peuplements d'algues photophiles de l'infralittoral. GIS Posidonie publication349-353.

- Verlaque M, Fritayre P (1994b) Mediterranean algal communities are changing in face of the invasive alga Caulerpa taxifolia (Vahl) C. Agardh. Oceanologia Acta 17: 659-672.

- Warwick RM, Clarke KR (1995) New biodiversity measures reveal a decrease in taxonomic distinctness with increasing stress. Marine Ecology Progress Series 129:

301-305. https://doi.org/10.3354/meps129301

- Warwick RM, Clarke KR (1998) Taxonomic distinctness and environmental assessment. Journal of Applied Ecology 35: 532-543. https://doi.org/10.1046/j. 1365-2664.1998.3540532.x

- Wilson O (1997) Foreword. In Strangers in Paradis. Impact and Management of nonindigenous species in Florida. Washington DC Island Press

\section{Supplementary materials}

\section{Suppl. material 1: average recovery: Salamandre Station doi}

Authors: Bachir Bouiadjra B., Ghellai M., Daoudi M., Behmene I.E., Bachir Bouiadjra M.A. Data type: Table.CSV

Brief description: average recovery by species and by season, Salamandre Station

Download file $(2.44 \mathrm{~kb})$ 


\section{Suppl. material 2: average recovery: Small Port Station doi}

Authors: Bachir Bouiadjra B., Ghellai M., Daoudi M., Behmene I.E., Bachir Bouiadjra M.A. Data type: Table.CSV

Brief description: average recovery by species and by season, Small Port Station Download file $(4.74 \mathrm{~kb})$

\section{Suppl. material 3: taxonomic classification of the species recorded doi}

Authors: Bachir Bouiadjra B., Ghellai M., Daoudi M., Behmene I.E., Bachir Bouiadjra M.A. Data type: table.CSV

Brief description: the taxonomic classification table, based on the phylogenetic classification, used for the calculation of taxonomic indices

Download file $(10.86 \mathrm{~kb})$ 